\title{
In vitro Antimicrobial Activity of the Aminoglycoside Arbekacin Tested Against Oxacillin-Resistant Staphylococcus aureus Isolated in Brazilian Hospitals
}

Julio C. R. Cordeiro, Adriana O. Reis, Eliete A. Miranda, Helio S. Sader and The Arbekacin Study Group

\author{
Special Clinical Microbiology Laboratory, Division \\ of Infectious Diseases, Universidade Federal de São \\ Paulo, SP, Brazil
}

\begin{abstract}
Arbekacin is an aminoglycoside used in Japan for treating infections caused by gentamicin and oxacillin-resistant $S$. aureus (ORSA). The objective of this study was to determine the in vitro antimicrobial activity of arbekacin against 454 clinical isolates of ORSA. The isolates were consecutively collected between January and July, 2000, from patients hospitalized in 8 Brazilian medical centers. The antimicrobial susceptibility testing was performed by disk diffusion method according to NCCLS recommendations. The vast majority of the isolates, 453 strains $(\mathbf{9 9 . 8 \%})$, were considered susceptible to arbekacin based on the criteria proposed by the Requirements for Antibiotic Products of Japan. Only 1 isolate (0.2\%) was classified as resistant. On the other hand, high rates of resistance were demonstrated for other aminoglycosides, such as gentamicin (97.6\% resistance) and amikacin $(\mathbf{9 7 . 0 \%}$ resistance). Resistance rate was also high for ciprofloxacin $(98.0 \%)$. All isolates were considered susceptible to vancomycin. The excellent in vitro antimicrobial activity of arbekacin demonstrated in this study indicates that this antimicrobial agent may play an important role in the treatment of severe ORSA infections, especially those that show poor clinical response with vancomycin monotherapy. Since the aminoglycosides should not be used as monotherapy to treat Gram positive infections, further studies evaluating in vitro and in vivo synergistic activity of arbekacin combinations are necessary to clarify the clinical role of this aminoglycoside.

Key Words: Staphylococcus aureus, arbekacin, oxacillin-resistant, ORSA, antimicrobial resistance, nosocomial Infections.
\end{abstract}

Infections due to ORSA strains represent a serious clinical and epidemiologic problem, especially among seriously ill inpatients [1]. Most strains of ORSA exhibit high-level resistance to most commercially available antimicrobial agents. Vancomycin has been the main antimicrobial agent

Received on 17 February 2001; revised 18 April 2001.

Address for correspondence: Dr. Prof. Dr. Hélio S. Sader. Laboratório Especial de Microbiologia Clínica - Disciplina de Doenças Infecciosas e Parasitárias. Universidade Federal de São Paulo - EPM. Rua Botucatu, 740. São Paulo, SP, Zip Code: 04023-063 -Brazil. Phone: (55 11) 5081-2819/5571-5180/55764393. Fax:(55 11) 5543-3013/5571-5180.

E-mail: heliosader@uol.com.br

The Brazilian Journal of Infectious Diseases 2001;5(3):130-135 (C) 2001 by The Brazilian Journal of Infectious Diseases and Contexto Publishing. All rights reserved.

$1413-8670$ for treating infections caused by ORSA $[1,2]$. However, S. aureus with intermediate resistance to glycopeptides has been reported in Japan, USA, France, and Hong Kong [3,4]. Although the level of resistance is low, treatment with vancomycin failed in most of the reported cases, raising concerns that treatment of ORSA infections may become even more problematic in the future [3, 4].

The vast majority of $S$. aureus strains with reduced susceptibilities to vancomycin, described until now have initially been ORSA strains that developed decreased susceptibility to vancomycin after prolonged exposure to this compound $[3,4]$. The prolonged use of vancomycin will likely lead to additional resistance by Gram positive organisms. Therefore, the potential for continuing emergence of $S$. aureus strains with intermediate resistance to vancomycin increases the need for new therapeutic options [5]. 
Aminoglycosides have been used to treat bacterial infections for a long time [1]. The critical resistance mechanisms to aminoglycoside antibiotics in bacteria of clinical importance are the enzymatic $N$-acetylation, $O$-nucleotidylation and $O$-phosphorylation, that generally results in the inactivation of these compounds [6]. The genes for these aminoglycoside-modifying enzymes are located mainly on plasmids [7]. Organisms expressing resistance due to decreased aminoglycoside permeability have also been reported, and resistance due to altered target (ribosome modifications) is rare among clinical isolates [6]. Studies of the resistance mechanism and chemical modifications of aminoglycoside antibiotics led to the synthesis of arbekacin in 1973, from the $1-N$-acylation of dibekacin with (S)-4-amino-2-hydroxybutyric acid [6]. Arbekacin is an aminoglycoside with broad spectrum of antimicrobial activity, which includes bacteria resistant to kanamycin, gentamicin and tobramycin [2,6]. Arbekacin is active in vitro against both Gram negative and Gram positive aerobic bacteria, and it is refractory to hydrolysis by most aminoglycoside modifying enzymes produced by aerobic bacteria [6].

Arbekacin was introduced in Japan in the late 1990s as a useful chemotherapeutic agent for the treatment of infections caused by ORSA [8]. Arbekacin acts by binding to both $50 \mathrm{~S}$ and $30 \mathrm{~S}$ ribosomal sub-units. Thus, it inhibits protein synthesis at bacterial ribosomes and causes codon misreading $[6,9]$. The high stability of arbekacin in the presence of inactivating enzymes produced by ORSA, such as 2"-aminoglycoside-phosphotransferase, 4"aminoglycoside-adenylyltransferase and3"-aminoglycosidephosphotransferase causes gentamicin-resistant ORSA strains to be susceptible to this antimicrobial agent $[6,10]$.

The objective of this study is to determine the in vitro antimicrobial activity profile of arbekacin against clinical ORSA isolates collected in Brazilian medical centers.

\section{Materials and Methods}

\section{Bacterial samples}

Four hundred fifty four clinical ORSA isolates were included in the study. The isolates were consecutively collected between January to July, 2000, in 8 Brazilian medical centers. The following centers were included in the study: Laboratório Especial de Microbiologia Clínica - Hospital São Paulo (LEMC), São Paulo (126 strains); Laboratório de Investigações Médicas da Universidade de São Paulo (LIM-54), São Paulo (60 strains); Hospital de Base de Brasília (HBB), Distrito Federal (85 strains); Hospital de Clínicas de Porto Alegre (HCPA), Rio Grande do Sul (50 strains); Laboratório Médico Santa Luzia (LMSL), Florianópolis, Santa Catarina (45 strains); Messejana Hospital (MH), Fortaleza, Ceará (34 strains); Real Hospital Beneficiência Portuguêsa (RHBP), Recife, Pernambuco (30 strains); and Hospital Universitário da Universidade Federal do Rio de Janeiro (HUUFRJ), Rio de Janeiro (24 strains). Two other medical centers (Emílio Ribas Hospital, São Paulo, and Laboratório Médico Jorge Saliba, Belo Horizonte, Minas Gerais) participated in the study; however, their results were excluded from the analyses because the strains were not saved for re-testing and/or confirmation of the initial results.

\section{Antimicrobial susceptibility tests}

The samples were evaluated using the disk diffusion method according to standards of the National Committee for Clinical Laboratory Standards (NCCLS) [11]. The susceptibility tests were performed by each participanting center. The antimicrobial agents evaluated were as follows: arbekacin (30 mg disk), vancomycin (30 mg disk), oxacillin (1 mg disk), amikacin (30 mg disk), ciprofloxacin (5 mg disk), and gentamicin (10 mg disk). All isolates with reduced susceptibility to arbekacin (inhibition zone $\leq 20 \mathrm{~mm}$ ) and/or vancomycin were sent to the coordinator center for retest. The isolates were categorized as susceptible, intermediate, and resistant according to the standards established by the NCCLS [11]. The interpretation criteria used for arbekacin were based on the Requirements for Antibiotic Products of Japan, that is cited in its package insert [12]. This document classifies the isolates as follows: susceptible when inhibition zone diameter is $\geq 21 \mathrm{~mm}$, moderately susceptible when 
Figure 1. Distribution of arbekacin zone diameters for 454 oxacillin-resistant Staphylococcus aureus (ORSA) isolates

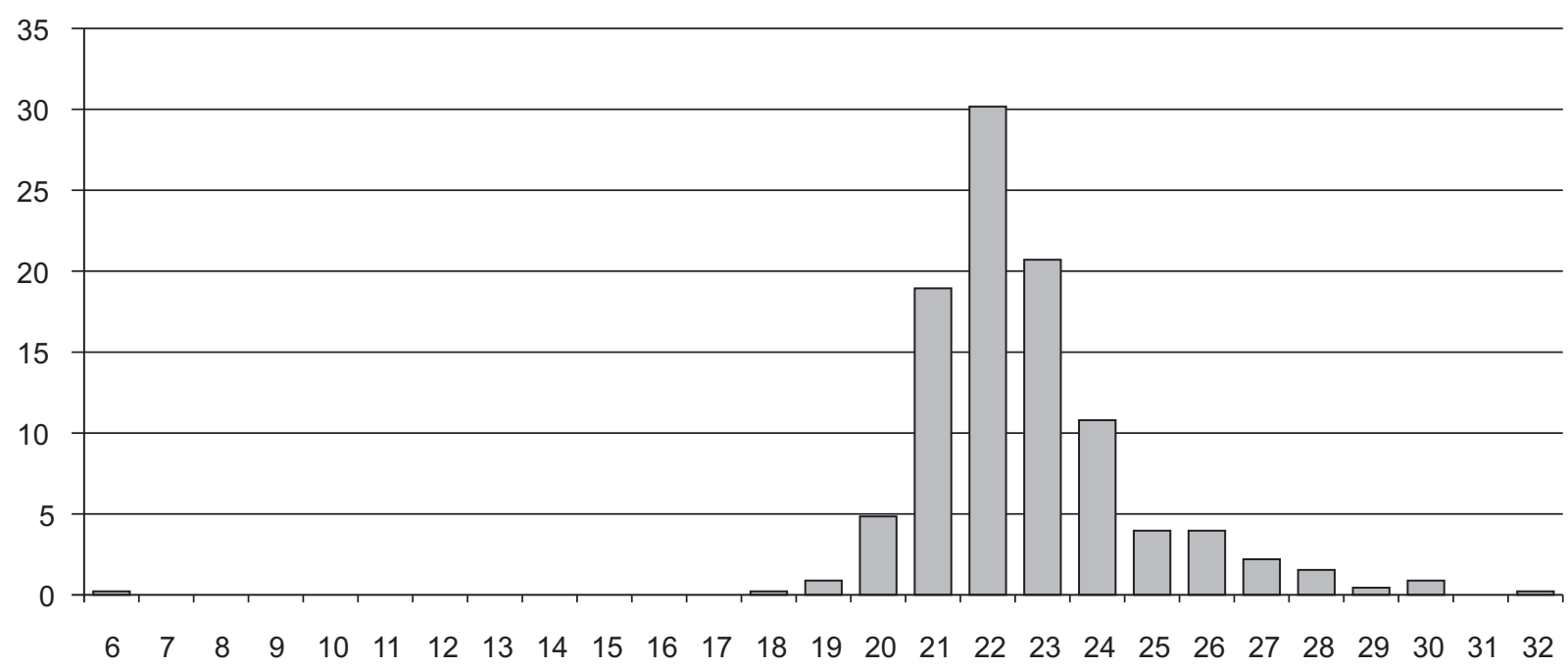

Table 1. Antimicrobial susceptibility pattern of ORSA strains from Brazilian hospitals

\begin{tabular}{lccc}
\hline Antimicrobial agents & \% Resistant & \% Intermediate & \% Susceptible \\
\hline Arbekacin & 0.2 & 0.0 & 99.8 \\
Gentamicin & 97.6 & 1.3 & 1.1 \\
Amikacin & 97.0 & 0.4 & 2.6 \\
Ciprofloxacin & 98.0 & 0.2 & 1.8 \\
Oxacillin & 100.0 & 0.0 & 0.0 \\
Vancomycin & 0.0 & 0.0 & 100.0 \\
\hline
\end{tabular}

Figure 2. ORSA isolate showing in vitro susceptibility only to arbekacin and vancomycin

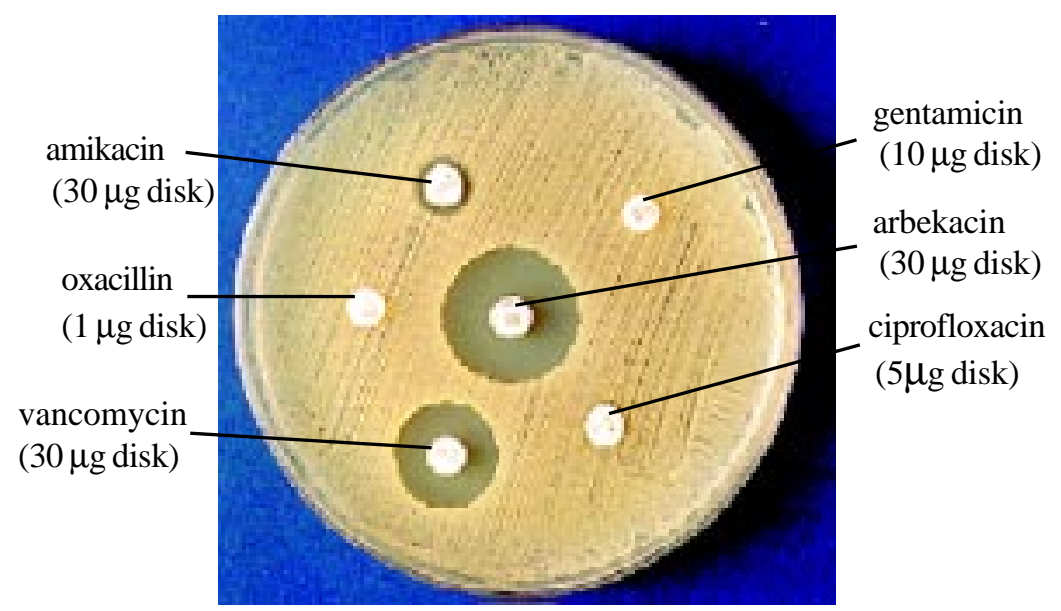


Table 2. Antimicrobial susceptibility pattern of ORSA strains according to the participating centers

\begin{tabular}{lcccc}
\hline Center (n) & \multicolumn{4}{c}{ \% Susceptible Strains } \\
\cline { 2 - 5 } & Arbekacin & Gentamicin & Ciprofloxacin & Vancomycin \\
\cline { 2 - 5 } LEMC (126) & 99.2 & 2.4 & 2.4 & 100.0 \\
LIM-54 (60) & 100.0 & 0.0 & 0.0 & 100.0 \\
HBB (85) & 100.0 & 0.0 & 0.0 & 100.0 \\
HCPA (50) & 100.0 & 2.0 & 2.0 & 100.0 \\
LMSL (45) & 100.0 & 0.0 & 0.0 & 100.0 \\
MH (34) & 100.0 & 0.0 & 8.8 & 100.0 \\
RHBP(30) & 100.0 & 0.0 & 0.0 & 100.0 \\
HU-UFRJ (24) & 100.0 & 4.2 & 4.2 & 100.0 \\
\hline Total (454) & $\mathbf{9 9 . 8}$ & $\mathbf{1 . 1}$ & $\mathbf{1 . 8}$ & $\mathbf{1 0 0 . 0}$ \\
\hline
\end{tabular}

LEMC: Laboratório Especial de Microbiologia Clínica - Hospital São Paulo; LIM-54: Laboratório de Investigações Médicas da Universidade de São Paulo; HBB: Hospital de Base de Brasília; HCPA: Hospital de Clínicas de Porto Alegre; LMSL: Laboratório Médico Santa Luzia; MH: Messejana Hospital; RHBP: Real Hospital Beneficiência Portuguesa; HU-UFRJ: Hospital Universitário da Universidade Federal do Rio de Janeiro.

inhibition zone diameter is $16 \mathrm{~mm}-20 \mathrm{~mm}$, moderately resistant when inhibition zone diameter is $10 \mathrm{~mm}-15 \mathrm{~mm}$, and totally resistant when zone diameter is $\leq 9 \mathrm{~mm}$. $S$ aureus ATCC 25923 was tested along with each series of susceptibility tests as quality control [13].

\section{Results}

The antimicrobial susceptibility patterns of the isolates for arbekacin and 5 other antimicrobial agents are shown in Table 1. Based on the Requirements for Antibiotic Products of Japan [12] only 1 isolate $(0.2 \%)$ was considered resistant to arbekacin. Twenty-seven isolates (6.0\%) had arbekacin inhibition zones between $18 \mathrm{~mm}$ and $20 \mathrm{~mm}$ of diameter (Figure 1). These isolates are considered moderately susceptible based on the criteria used in Japan. However, the analysis of the distribution of inhibition diameter zones (Figure 1) indicates that these isolates are included in the normal population. Thus, these strains were considered susceptible to arbekacin. Also, the collection showed very high rates of resistance to other antimicrobial agents evaluated: $97.6 \%$ gentamicin resistance, $97.0 \%$ amikacin resistance, and $98.0 \%$ ciprofloxacin resistance. One of these ORSA isolates showing susceptibility only to arbekacin and vancomycin is shown in Figure 2.

The only arbekacin-resistant strain detected in the study was isolated in a patient hospitalized at the Hospital São Paulo. The moderately susceptible strains were isolated in the Laboratório Especial de Microbiologia Clínica (10 isolates), Laboratório de Investigações Médicas da Universidade de São Paulo (11 isolates); Hospital de Clínicas de Porto Alegre (3 isolates); Real Hospital Beneficiência Portuguêsa (2 isolates), Hospital Universitário da Universidade Federal do Rio de Janeiro (1 isolate). Three participant centers did not detect any isolate moderately susceptible to arbekacin: Hospital de Base de Brasília, Laboratório Médico Santa Luzia, and Messejana Hospital.

\section{Discussion}

Staphylococcus aureus, especially oxacillinresistant S. aureus (ORSA) has become a major cause of nosocomial infections [14]. In Brazil, the vast majority of ORSA isolates show cross-resistance to other 
antimicrobial agents, including other $\beta$-lactams, macrolides, clindamycin, ciprofloxacin, trimethoprim/ sulfamethozaxole, and aminoglycosides, leaving very few therapeutic options to treat infections caused by this microorganism [15]. The high prevalence of crossresistance to other antimicrobial agents is related to the dissemination of transposons with insertion sequences in the 50-kb mec region gene [16,17]. For example, the transposon Tn554 contains the ermA gene, which encodes inducible erythromycin resistance, and the IS431, an insertion sequence associated with resistance determinants is extremely common. The ability of IS431 elements through homologous recombination to trap and cluster resistance determinants with similar IS elements explains the multiple drug resistance phenotype that is characteristic of ORSA strains $[16,17]$.

This high level of resistance among ORSA strains is of great concern $[3,4]$. Therefore, there is an urgent need for alternative therapeutic options for the treatment of infections caused by this microorganism [4]. Arbekacin, an aminoglycoside synthesized in Japan, has been evaluated in several comparative studies to define its clinical efficacy and its synergistic effect with other antimicrobial agents, such as vancomycin and ampicillin-sulbactam [2,8,18-20]. Arbekacin-resistant mutants can be selected at a frequency of $10^{-4}$ to $10^{-5}$ among gentamicin-resistant ORSA strains by exposure to subinhibitory concentrations of arbekacin $[21,22]$. The arbekacin-resistant mutants may express a bifunctional enzyme, $\mathrm{AAC}\left(6^{\prime}\right) / \mathrm{APH}\left(2^{\prime \prime}\right)$, that is able to modify arbekacin [21, 22]. However, further studies are necessary to evaluate the resistance mechanism present in the strain detected in the present study.

Since arkekacin is commercially available in very few countries, its breakpoints for disk diffusion have not been widely evaluated. The analysis of population distribution according to arbekacin inhibition diameter zone clearly shows that isolates classified as "moderately susceptible" (inhibition zones of $16 \mathrm{~mm}$ to $20 \mathrm{~mm}$ ) by the Requirements for Antibiotic Products of Japan are within the normal population and should be considered susceptible (Figure 1). Further studies evaluating the arbekacin minimum inhibitory concentration (MIC) of these strains are necessary to clarify the in vitro activity of arbekacin against these strains. On the other hand, the isolate with an inhibition zone of $6 \mathrm{~mm}$ is clearly out of the normal population and has probably acquired a mechanism of resistance to this new aminoglycoside.

The excellent in vitro activity showed in this first Brazilian benchmark study, coupled with the therapeutic efficacy demonstrated in treating infections caused by ORSA and GISA isolates in Japan, indicates that arbekacin may be a reasonable alternative for the treatment of ORSA and GISA infections in association with other anti-staphylococcal drugs [4, 18]. Further studies evaluating its antimicrobial potency and synergistic effect with other compounds will contribute to clarify the clinical role of this antimicrobial agent.

\section{Acknowledgements}

The authors would like to thank to Ana C. Gales, Rodrigo E. Mendes, and Amanda C. Lopes, for their important contributions to this study. This study received financial support from Eurofarma Laboratórios Ltda., São Paulo, Brazil.

\section{References}

1. Chambers F. H. Methicillin resistance in Staphylococci: Molecular and Biochemical basis and clinical implications. Clin Microbiol Rev 1997;10:781-91.

2. You I., Kariyama R., Zervos J. M., Kumon H., Chow W. J. In vitro activity of arbekacin alone and in combination with vancomycin against gentamycin- and methicillinresistant Staphylococcus aureus. Diag Microbiol Infect Dis 2000;36:37-41.

3. Tenover C. F., Lancaster M. V., Hill B. C., Hiramatsu K. Characterization of staphylococci with reduced susceptibilities to vancomycin and other glycopeptides. J Clin Microbiol 1998;36:1020-7.

4. Hiramatsu K., Hanaki H., Tenover F. C. et al. Methicillinresistant Staphylococcus aureus clinical strain with reduced vancomycin susceptibility. J Antimicrob Chemother 1997; 40:135-46.

5. Akins L. M., Rybak J. M. In vitro activities of daptomycin, arbekacin, vancomycin, and gentamicin alone and/or in combination against glycopeptide intermediateresistant Staphylococcus aureus in an infection model. Antimicrob Agents Chemother 2000;44:1925-9. 
6. Kondo S., Hotta K. Semisynthetic aminoglycoside antibiotics: Development and enzymatic modifications. J Infect Chemother 1999;5:1-9.

7. Davies J., Smith D. I. Plasmid-determined resistance to antimicrobial agents. Annu Rev Microbiol 1987; 32:469-518.

8. Inoue M., Nonoyama M., Okamoto R., Ida T. Antimicrobial activity of arbekacin, a new aminoglycoside antibiotic, against methicilin-resistant Staphylococcus aureus. Drugs Exp Clin Res 1994;20:233-9.

9. Watanabe T., Ohashi K., Matsui K., Kubota T. Comparative studies of the bactericidal, morphological and post-antibiotic effects of arbekacin and vancomycin against methicillin resistant Staphylococcus aureus. J Antimicrob Chemother 1997;39:471-6.

10. Kondo S., Tamura A., Gomi S.,et al. Structures of enzymatically modified products of arbekacin by methicillin-resistant Staphylococcus aureus. J Antibiotics 1993;46:310-5.

11. National Committee for Clinical Laboratory Standards (NCCLS). Performance standards for antimicrobial disk susceptibility tests: approved standard M2-A6. Sixth edition. Wayne, PA, 1997.

12. Arbekacin Showa Disc (package insert). Bacterial sensitivity test drug. Showa Yakuhin Kako Co. Ltd. 1718, Kyobashi 2-chome, Chuo-ku, Tokyo 104, Japan.

13. National Committee for Clinical Laboratory standards (NCCLS). Performance standards for antimicrobial susceptibility testing. Ten informational supplements. NCCLS document M100-S10. Wayne, PA, 2000.

14. Lelievre H., Gerard L., Jones E. M. et al. Emergence and spread in French hospitals of methicillin-resistant Staphylococcus aureus with increasing susceptibility to gentamicin and other antibiotics. J Clin Microbiol 1999;37:3452-7.

15. Teixeira L. A., Resende C. A., Ormonde L. R. Geographic spread of epidemic multiresistant Staphylococcus aureus clone in Brazil. J Clin Microbiol 1995;33:2400-4.

16. Archer G. L., Niemeyer D. M. Origin and evolution of DNA associated with resistance to methicillin in Staphylococci. Trends Microbiol 1994,2:343-7.

17. Matthews P. R., Reed K. C., Stewart P. R. The cloning of chromosomal DNA associated with methicillin and other resistances in Staphylococcus aureus. J Gen Microbiol 1987;133:1919-29.

18. Kono K., Takeda S., Tatara I., Arakawa K. In vitro activities of arbekacin, alone and in combination, against methicillin-resistant Staphylococcus aureus. Jap J Antib 1994;46:710-9.

19 Deguchi K., Yokota N., Kogushi M. et. al. Antibacterial activities of arbekacin against recently isolated methicillin-resistant Staphylococcus aureus. Jap J Antib 1993;46:234-41.
20. Morimoto K., Nakatami S., Kaji M., Kinoshita H. Treatment with arbekacin of surgical infections by resistant strains of Staphylococcus aureus. Arbekacin Study Group. Jap J Antib 1994;47:826-36.

21. Obayashi Y., Fujita J., Ichyiama S., et al. Investigation of nosocomial infection caused by arbekacin-resistant, methicillin-resistant Staphylococcus aureus. Diag Microb Infec Dis 1997;28:53-9.

22. Suzuki T., Fujita K., Nagamachi Y. Emergence of arbekacinresistant strains among methicillin-resistant Staphylococcus aureus. Jap J Antib 1994;47:634-9. 